http://dx.doi.org/10.35381/s.v.v5i9.1148

\title{
Actitud comunitaria en la prevención del contagio por COVID-19
}

Community attitude in the prevention of contagion by COVID-19

\author{
Narcisa Liliana Durán-Ávila \\ narcisa.duran@unesum.edu.ec \\ Universidad Estatal del Sur de Manabí, Jipijapa \\ Ecuador \\ https://orcid.org/0000-0002-6088-0551 \\ Virginia Esmeralda-Pincay \\ virginia.pincay@unesum.edu.ec \\ Universidad Estatal del Sur de Manabí, Jipijapa \\ https://orcid.org/0000-0001-8776-5433 \\ Ángel Luis Zamora-Cevallos \\ angelluisz2007@hotmail.com \\ Universidad Estatal del Sur de Manabí, Jipijapa \\ Ecuador \\ https://orcid.org/0000-0001-8547-3592 \\ Tania Mercedes Alcázar-Pichucho \\ tania.alcazar@unesum.edu.ec \\ Universidad Estatal del Sur de Manabí, Jipijapa \\ Ecuador \\ https://orcid.org/0000-0001-7440-5447
}

Recepción: 01 de octubre 2020

Revisado: 01 de noviembre 2020

Aprobación: 20 de diciembre 2020

Publicación: 01 de enero 2021 


\title{
RESUMEN
}

Objetivo: El estudio de la actitud comunitaria en la prevención del contagio por COVID 19 del Cantón de Jipijapa. Metodología: de enfoque cuantitativo y una tipología descriptiva con un diseño no experimental, de campo y transaccional. La población la constituyeron 250 habitantes de las comunidades del Cantón de Jipijapa. Conclusión: Fue posible evidenciar que un alto porcentaje de las personas investigadas asumen una actitud comunitaria asociada al cumplimiento de las medidas de prevención implementadas por el sistema de salud y la región, lo cual puede verse entorpecido por la actitud individual de los habitantes, dado el desconocimiento de la evolución de la epidemia y su incidencia en los grupos vulnerables de las comunidades afectadas.

Descriptores: Infecciones por coronavirus; salud mental; depresión. (Fuente: DeCS).

\begin{abstract}
Objective: The study of community attitude in the prevention of contagion by COVID -19 of the Canton of Jipijapa. Methodology: Quantitative approach and descriptive typology with a non-experimental, field and transactional design. The population was made up of 250 inhabitants of the communities of the Canton of Jipijapa. Conclusion: critical functions such as community attitude, protection of vulnerable groups, support for health workers, case detection, contact tracing and compliance with physical estating measures can only be given with the support of each and every person in the affected communities.
\end{abstract}

Descriptors: Coronavirus infections; mental health; depression. (Source: DeCS). 
Narcisa Liliana Durán-Ávila; Virginia Esmeralda-Pincay; Ángel Luis Zamora-Cevallos; Tania Mercedes Alcázar-Pichucho

\section{INTRODUCCION}

Las autoridades sanitarias están advirtiendo que la evolución de la epidemia de coronavirus dependerá de forma crucial del comportamiento individual y comunitario de las personas. El comportamiento de los seres humanos ante una epidemia, como la actual de Covid-19, tiende a ser "irracional" ${ }^{1}$. En este sentido, las funciones críticas, como la actitud comunitaria, la protección de los grupos vulnerables, el apoyo a los trabajadores sanitarios, la detección de casos, el rastreo de contactos y el cumplimiento de las medidas de distanciamiento físico solo pueden darse con el apoyo de todas y cada una de las partes de las comunidades afectadas. Por ello, el empoderarse de las comunidades es la vía para garantizar que los servicios y la ayuda se planifiquen y adapten en función de su opinión y contexto locales².

Cabe destacar, que el comportamiento comunitario ${ }^{3}$, apunta a tres temas principales, provenientes de la Psicología, que ha incorporado la economía del comportamiento al estudio de las decisiones económicas que están presentes en la crisis epidemiológica del Covid-19.

En primer término, habla de los resultados inciertos, por desconocimiento del origen y de la evolución de la epidemia, la efectividad de las medidas de contención implementadas, la posibilidad de tener acceso a la vacuna en un futuro cercano o cómo el comportamiento individual y colectivo puede afectar realmente a la probabilidad de contagio. Ante la existencia de incertidumbre, la heterogeneidad en la actitud individual ante el riesgo dificulta la coordinación de las mejores medidas preventivas y aceptar las recomendaciones de las autoridades favoreciendo la entrada del pánico, implicando el colapso sin necesidad de los centros médicos y desabasteciendo de recursos sanitarios a los que realmente los necesitan ${ }^{5}$.

En segundo término, cuando se da de forma individual y/o comunitario, mayor importancia al presente que el futuro, al igual que otros comportamientos saludables difíciles de conseguir es incentivar el comportamiento preventivo de la población ante una epidemia, específicamente en el aumentar la higiene, evitar acudir a eventos multitudinarios, hacer cuarentena ante la sospecha de infección, no colapsar 
urgencias por síntomas breves, no malgastar las mascarillas disponibles y necesarias para enfermos y profesionales sanitario ${ }^{3}$.

El tercer tema, es el comportamiento preventivo ante una epidemia que tiene un componente de bien público importante, tal es el caso de declarar el haber estado en una zona de riesgo, cancelar la participación en un evento de asistencia masiva, entendiendo que la ausencia de comportamientos individuales que favorezca al colectivo, incide en la extensión de la epidemia a las comunidades ${ }^{3}$. Toda esta problemática expuesta, pareciera estar presente entre los miembros de la comunidad del Cantón de Jipijapa, al observarse desconocimiento de la población en relación a la pandemia originada por el COVID-19, asimismo presentando una actitud de defensa individual sin considerar la de tipo comunitaria, aunado al poco comportamiento preventivo que imponen las autoridades sanitarias de la región ${ }^{4}$. Con base a esta situación, se plantea este artículo el estudio de la actitud comunitaria en la prevención del contagio por COVID -19 del Cantón de Jipijapa

Cada país debe ${ }^{6}$, seguir aplicando los planes de acción nacionales basados en un enfoque de la sociedad en su conjunto y una valoración realista de lo que es factible lograr en cuanto a la ralentización de la transmisión y la reducción de la mortalidad y, posteriormente, en cuanto al sostenimiento de un bajo nivel de transmisión mientras se reanuda la actividad social y económica. Se indica ${ }^{71}$ que se debe educar a la población para mejorar la prevención de infecciones relacionadas con la atención de la salud, solo así, es posible asegurar un progreso social por su trascendencia económica y un desafío para las instituciones de salud y para el personal responsable del cuidado. El objetivo primordial ${ }^{6}$ es que todos los países controlen la pandemia mediante la ralentización de la transmisión y la reducción de la mortalidad asociada a la COVID-19. 


\section{Participación comunitaria y su movilización para limitar la exposición.}

Frenar la transmisión de la COVID-19 y proteger las comunidades requerirá de la participación de todos los miembros de las comunidades ${ }^{612}$ en riesgo y afectadas para evitar el contagio y la transmisión. Esto exige que todos tomen medidas de protección individual como lavarse las manos, evitar tocarse la cara, practicar una buena higiene respiratoria, distanciamiento a nivel individual y cooperar con las medidas de distanciamiento físico y de restricción de movimiento cuando se solicite adoptarlas ${ }^{9}$. Por lo tanto, es fundamental que las autoridades internacionales, nacionales y locales lleven a cabo acciones participativas de comunicación bidireccional de forma proactiva, regular, transparente y $\sin$ ambigüedades con todas las poblaciones afectadas o en riesgo.

El objetivo de la investigación fue analizar la Actitud comunitaria en la prevención del contagio por COVID -19 del Cantón de Jipijapa - Ecuador.

\section{METODOLOGIA}

\section{Tipo y diseño}

De tipo descriptiva observacional con diseño no experimental transaccional.

\section{Muestra poblacional}

Conformada por 250 personas que habitan en el Cantón Jipijapa - Provincia de Manabí - Ecuador.

\section{Criterios de inclusión de la muestra poblacional}

Pertenecer a alguna organización o institución de salud y estar trabajando en la prevención comunitaria del COVID-19. 


\section{Técnicas e instrumentos de investigación}

Se aplicó encuesta y un instrumento tipo cuestionario de preguntas semi estructuradas, el cual es ${ }^{8}$ el proceso que sigue el investigador para recolectar 0 conocer los datos y/o informaciones de una población investigada.

\section{Análisis de la información}

Se procesó mediante estadística descriptiva.

\section{RESULTADOS}

Posterior al análisis de la información obtenida y en contraste con las literaturas revisadas, se presentan los siguientes resultados:

\section{Tabla 1.}

Análisis descriptivo frecuencial del indicador cumplimiento de las medidas preventivas al COVID-19 en el Cantón de Jipijapa.

\begin{tabular}{lllll}
\hline $\begin{array}{l}\text { Análisis/ } \\
\text { indicador }\end{array}$ & Fr & $\%$ & FrA & $\% A$ \\
\hline Si cumplen & 189 & $\mathbf{7 5 , 6}$ & 189 & $\mathbf{7 5 , 6}$ \\
Parcial cumplimiento & 50 & 20 & 239 & 95,6 \\
No cumplen & 11 & 4,4 & 250 & 100 \\
\hline Total & 250 & 100 & 250 & 100 \\
\hline
\end{tabular}




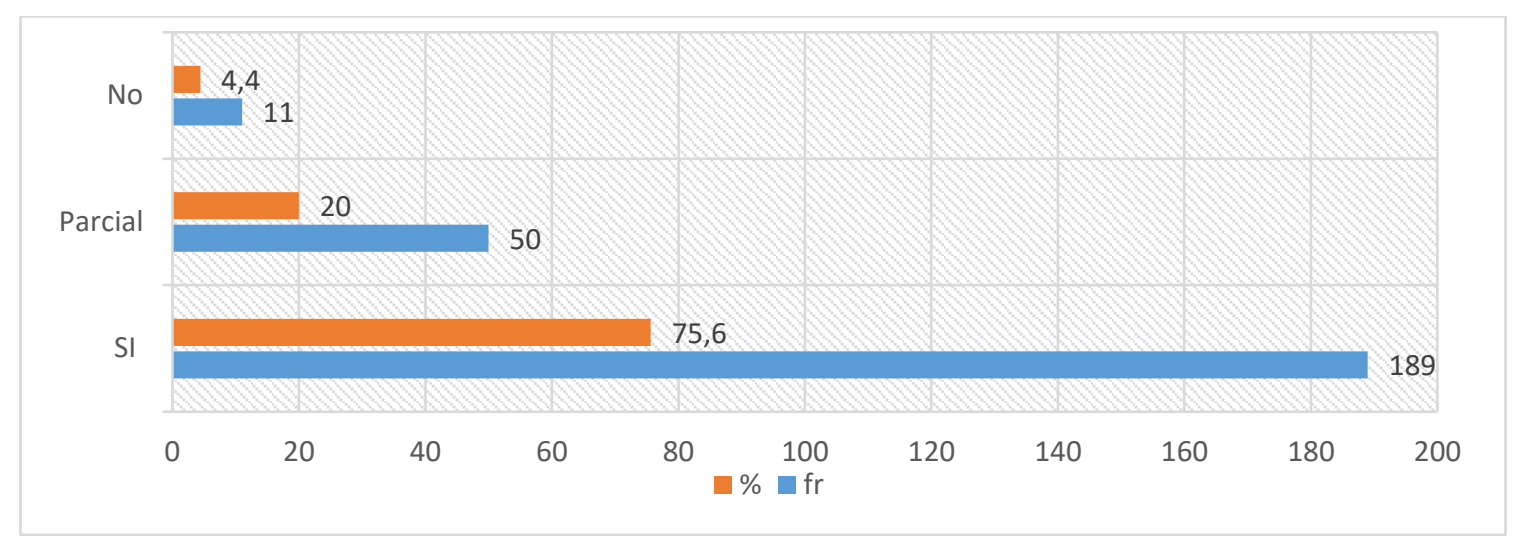

Grafico 1: Análisis descriptivo frecuencial del indicador cumplimiento de las medidas preventivas al COVID-19 en el Cantón de Jipijapa.

Se indicó que el $75.6 \%$ de los investigados coincidieron en opinar que si se cumplen las medidas de contención implementadas por el sistema de salud y la región, las cuales pueden verse entorpecidas por la actitud individual de los habitantes, dado el desconocimiento del origen y de la evolución de la epidemia, la efectividad. Seguido de la alternativa parcialmente se cumple con el $20 \%$ de las opiniones, el menor porcentaje con el $4,4 \%$ indicó la alternativa no se cumplen.

\section{Tabla 2}

Análisis descriptivo frecuencial del indicador importancia a la información en redes sociales acerca de la pandemia del COVID-19 en el Cantón de Jipijapa.

\begin{tabular}{lllll}
\hline $\begin{array}{l}\text { Análisis/ } \\
\text { indicador }\end{array}$ & Fr & $\%$ & FrA & $\% A$ \\
\hline Muy importante & 167 & 67 & 167 & 67 \\
Mediana importancia & 83 & 33 & 250 & 100 \\
No importante & 0 & 4,4 & 250 & 100 \\
\hline Total & $\mathbf{2 5 0}$ & $\mathbf{1 0 0}$ & $\mathbf{2 5 0}$ & $\mathbf{1 0 0}$ \\
\hline
\end{tabular}




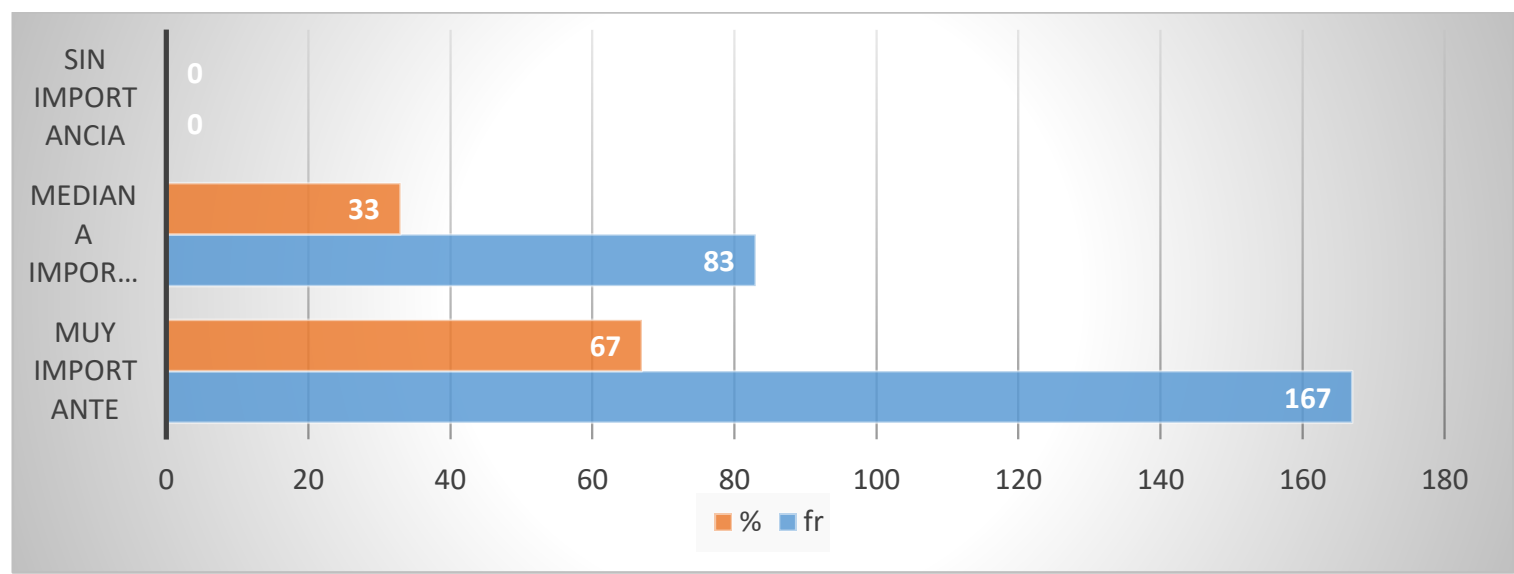

Grafico 2: Análisis descriptivo frecuencial del indicador importancia a las medidas preventivas al COVID-19 en el Cantón de Jipijapa.

El $67 \%$ de los investigados indicaron mediana importancia a la información relacionada a la pandemia por las redes sociales que desfavorece el incentivo a un comportamiento preventivo de la población ante una epidemia. El resto representado por el $33 \%$ indico que es muy importante y necesario no considerar aquellas informaciones que carecen de sustento científico y veraz para prevenir la enfermedad. Estos resultados difieren de lo propuesto por la OMS ${ }^{6}$, quienes afirman que cada región debe tener planes de acción informativos y concretos basados en un enfoque de la sociedad que permita sostener un bajo nivel de transmisión mientras se reanuda la actividad social y económica.

\section{Tabla 3}

Análisis descriptivo frecuencial del indicador cumplimiento del uso y prácticas del protocolo para la prevención del COVID-19 en el Cantón de Jipijapa.

\begin{tabular}{lllll}
\hline $\begin{array}{l}\text { Análisis/ } \\
\text { indicador }\end{array}$ & Fr & $\%$ & FrA & $\% A$ \\
\hline Total cumplimiento & $\mathbf{1 7 7}$ & $\mathbf{7 1}$ & $\mathbf{1 7 7}$ & $\mathbf{7 1}$ \\
Parcial cumplimiento & $\mathbf{7 3}$ & $\mathbf{2 9}$ & $\mathbf{2 5 0}$ & $\mathbf{1 0 0}$ \\
No cumplimiento & $\mathbf{0}$ & $\mathbf{0}$ & $\mathbf{2 5 0}$ & $\mathbf{1 0 0}$ \\
\hline Total & $\mathbf{2 5 0}$ & $\mathbf{1 0 0}$ & $\mathbf{2 5 0}$ & $\mathbf{1 0 0}$ \\
\hline
\end{tabular}




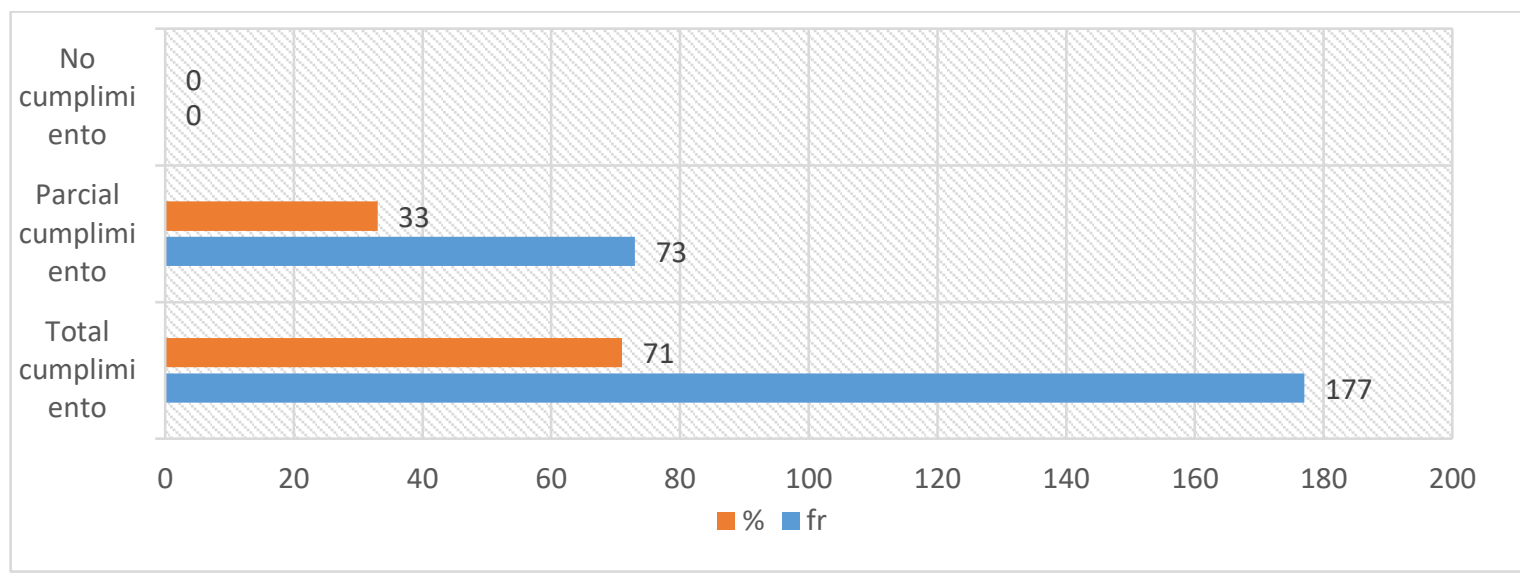

Grafico 3: Análisis descriptivo frecuencial del indicador cumplimiento del uso y prácticas del protocolo para la prevención del COVID-19 en el Cantón de Jipijapa.

El 71\% índico que entre las medidas de prevención hacen uso de mascarillas, respeto la distancia y lavados de manos como medidas de prevención a la pandemia fuera del hogar $y / 0$ al trasladarse a sus trabajos o zonas cercanas a su vivienda, mientras que el 29 \% lo hacen parcialmente. Se destaca el hecho en la cual no hubo opinión alguna para la alternativa no utilizo medios de prevención. Lo datos expuestos permiten reforzar la necesidad reforzar y ejecutar la propuesta de la OMS ${ }^{6}{ }^{13}$, en relación con la participación comunitaria y su movilización para limitar la exposición, y de esta manera frenar la transmisión de la COVID-19 y proteger a las comunidades evitando su contagio y transmisión. Esto exige que todos tomen medidas de protección individual como lavarse las manos, evitar tocarse la cara, practicar una buena higiene respiratoria, distanciamiento a nivel individual y cooperar con las medidas de distanciamiento físico y de restricción de movimiento cuando se solicite adoptarlas ${ }^{10}$ 1415 . 
Narcisa Liliana Durán-Ávila; Virginia Esmeralda-Pincay; Ángel Luis Zamora-Cevallos;

Tania Mercedes Alcázar-Pichucho

Tabla 4.

Análisis descriptivo frecuencial del indicador actitud individual en el cumplimiento a las medidas preventivas al COVID-19 en el Cantón de Jipijapa.

\begin{tabular}{lllll}
\hline $\begin{array}{l}\text { Análisis/ } \\
\text { indicador }\end{array}$ & Fr & $\%$ & FrA & $\% A$ \\
\hline Total cumplimiento & 142 & $\mathbf{5 7}$ & $\mathbf{1 4 2}$ & $\mathbf{5 7}$ \\
Parcial cumplimiento & 95 & 38 & 237 & 95 \\
No cumplimiento & 13 & 5 & 250 & 100 \\
\hline Total & $\mathbf{2 5 0}$ & $\mathbf{1 0 0}$ & $\mathbf{2 5 0}$ & $\mathbf{1 0 0}$ \\
\hline
\end{tabular}

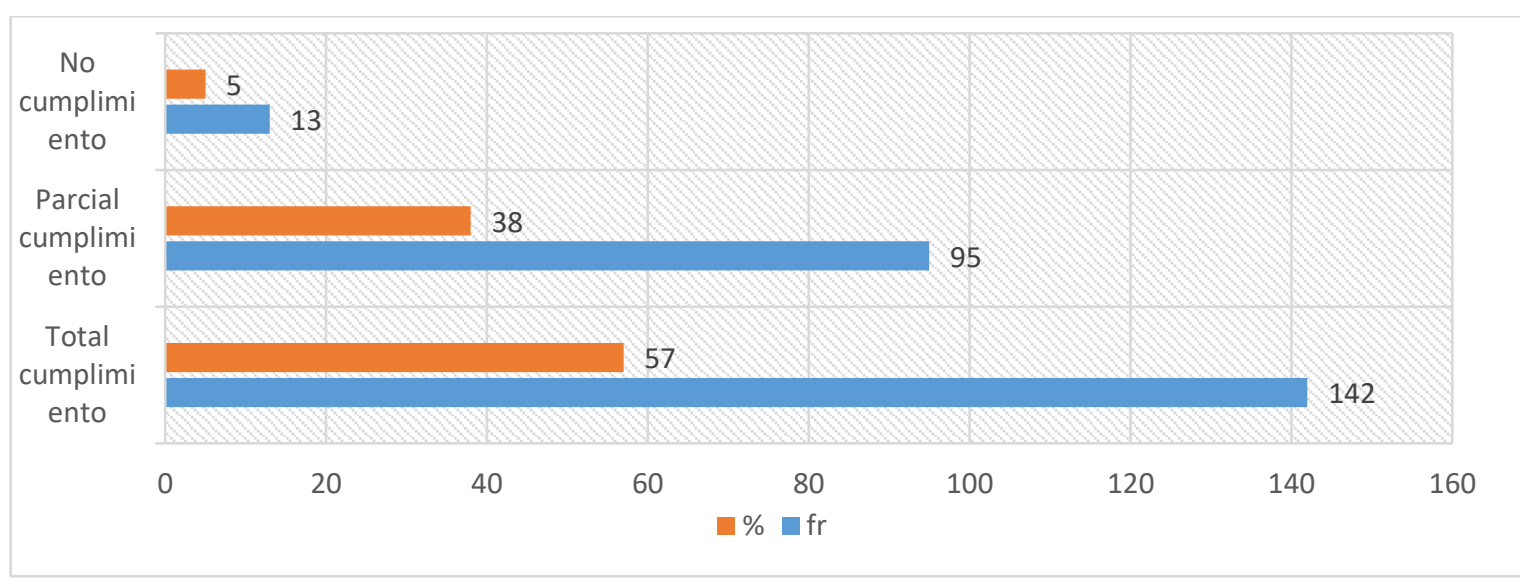

Grafico 4: Análisis descriptivo frecuencial del indicador actitud individual en el cumplimiento a las medidas preventivas al COVID-19 en el Cantón de Jipijapa.

El $57 \%$ de los habitantes considera necesario tener un comportamiento preventivo al declarar que se ha estado en una zona de riesgo y cancelar su participación en algún evento de asistencia masiva, logrando de esta forma privar el comportamiento individual para favorecer el colectivo. El $38 \%$ indico que solo algunas veces lo haría y el $5 \%$ opino de forma negativa. De allí, que es posible indicar que los efectos experimentados durante la Pandemia del COVID-19, han afectado con mayor 
frecuencia a las comunidades cuyos habitantes hacen caso omiso a las medidas de prevención.

\section{CONCLUSIONES}

Se evidenció que un alto porcentaje de las personas investigadas asumen una actitud comunitaria asociada al cumplimiento de las medidas de prevención implementadas por el sistema de salud y la región, lo cual puede verse entorpecido por la actitud individual de los habitantes, dado el desconocimiento de la evolución de la epidemia y su incidencia en los grupos vulnerables de las comunidades afectadas.

La comunidad de Jipijapa presta medianamente atención a las teorías de conspiración y desinformación con respecto al origen, la escala, la prevención, el tratamiento y otros aspectos de la enfermedad, que se viene dando después del brote inicial de la enfermedad del coronavirus 2019 (COVID-19), la cual se difunde a través de las redes sociales, mensajes de texto y algunos medios estatales en el país. También, se viene dando información médica errónea sobre formas de prevenir, tratar y auto diagnosticar la enfermedad y cuyas afirmaciones falsas pueden ser estafas comerciales que ofrecen pruebas en el hogar, supuestos preventivos y curas milagrosas

Es necesario concientizar la responsabilidad individual para frenar la expansión de una enfermedad colectiva como uno de los mayores retos a los que se enfrenta la humanidad en estos días, potenciando los comportamientos solidarios y comunitarios, que permita evadir el pánico o expandir información errónea, especialmente con el grado actual de conectividad que permiten las redes sociales.

Existe la necesidad de participación de toda la sociedad en los planes de coordinación nacional y subnacional, en pro de implantar de forma efectiva estrategias adaptativas de preparación y respuesta a la COVID-19 y de esta forma activarse mecanismos nacionales de gestión de emergencias de salud pública. 


\section{CONFLICTO DE INTERÉS}

Los autores declaran que no tienen conflicto de interés en la publicación de este artículo.

\section{FINANCIAMIENTO}

Autofinanciado.

\section{AGRADECIMIENTO}

A la Universidad Estatal del Sur de Manabí; por motivar el desarrollo de la investigación.

A la Comunidad del Cantón de Jipijapa; por su apoyo en el proceso investigativo.

\section{REFERENCIAS}

1. Segura MS. Con alerta, pero sin pánico. El rol de los medios durante la pandemia [Alerted but not panicked. The role of the media during the pandemic.]. Rev Fac Cien Med Univ Nac Cordoba. 2020 Mar 31;77(1):55-58. Spanish. doi: 10.31053/1853.0605.v77.n1.28066. PMID: 32238261.

2. Horowitz, J, Bubola, E y Povoledo, E. "Coronavirus: lecciones para el mundo desde Italia, el nuevo epicentro de la pandemia", The New York Times, 22 de marzo de 2020, https://n9.cl/fxy62

3. Nicola M, Alsafi Z, Sohrabi C, et al. The socio-economic implications of the coronavirus pandemic (COVID-19): A review. Int J Surg. 2020;78:185-193. doi:10.1016/j.ijsu.2020.04.018

4. Santos-Sanchez N, Salas-Coronado R. Origin, structural characteristics, prevention measures, diagnosis and potential drugs to prevent and COVID-19. Medwave 2020;20(8):e8037 doi: 10.5867/medwave.2020.08.8037

5. OMS. Organización Mundial para la Salud. Plan Estratégico de Preparación y Respuesta para la Enfermedad por Coronavirus 2019 (COVID-19). Pautas para la planificación operativa de la preparación y la respuesta de los países. https://n9.cl/yzxdz

6. OMS. Coronavirus: consejos de la OMS para prevenir el contagio. https://n9.cl/gfvye 
7. Banco Mundial de la Salud. Repuesta al Coronavirus. https://n9.cl/76dbx

8. Padua, J. Técnicas de investigación aplicada a las Ciencias sociales. Editorial Fondo Económico. México. 2012.

9. Ministerio de Salud de la Nación. COVID-19. Recomendaciones para Equipos de Salud. Recomendaciones para el Uso de los EPP. https://n9.cl/j9ohz

10. Tuaza Castro L. El COVID-19 en las comunidades indigenas de Chimborazo, Ecuador. Latin American and Caribbean Ethnic Studies, 2020:15:4, 413-424. https://doi.org/10.1080/17442222.2020.1829793

11. Burillo-Putze G, Espinosa Ramírez S, Lecuona Fernández M. Personal protection against COVID-19 in the emergency department: neither heroes nor cowards. Protección del personal de urgencias y emergencias frente al COVID19. Emergencias. 2020;32(4):223-224.

12. Sedano-Chiroque Franshesca L., Rojas-Miliano Cristhian, Vela-Ruiz José M. COVID-19 desde la perspectiva de la prevención primaria. Rev. Fac. Med. Hum. [Internet]. 2020; 20(3): 494-501.

13.Zhao Guanlan. Tomar medidas preventivas inmediatamente: evidencia de China sobre el COVID-19. Gac Sanit [Internet]. 2020; 34(3): 217-219.

14. Urzúa A, Vera-Villarroel $P$, Caqueo-Urízar A, Polanco-Carrasco R. La Psicología en la prevención y manejo del COVID-19. Aportes desde la evidencia inicial. TEPS [Internet]. 2020;38(1):103-18.

15. Candelaria Brito Julio Cesar, Díaz Cruz Sergio Alberto, Acosta Pérez Dulce María, Labrador Mazón Orestes, Rodríguez Méndez Alina. Estrategia intervencionista dirigida a la prevención y control de la COVID-19 en Consolación del Sur. Rev Ciencias Médicas [Internet]. 2020; 24(3): e4495. 